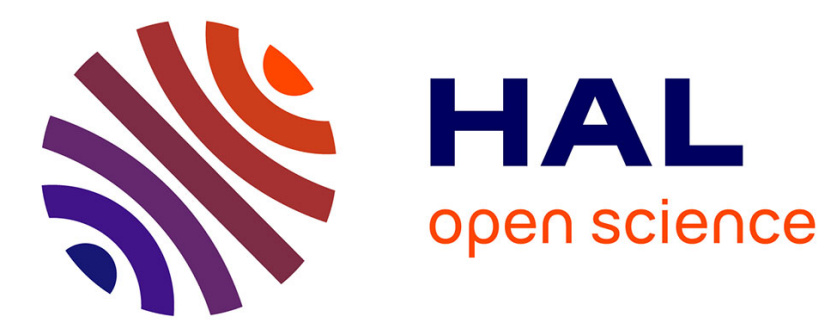

\title{
DEFECTS IN CRYSTALS CREATED BY ION BOMBARDMENT: THEORY AND SIMULATION
}

\author{
I. Torrens
}

\section{To cite this version:}

I. Torrens. DEFECTS IN CRYSTALS CREATED BY ION BOMBARDMENT : THEORY AND SIMULATION. Journal de Physique Colloques, 1973, 34 (C5), pp.C5-1-C5-7. 10.1051/jphyscol:1973501 . jpa-00215288

\section{HAL Id: jpa-00215288 https://hal.science/jpa-00215288}

Submitted on 1 Jan 1973

HAL is a multi-disciplinary open access archive for the deposit and dissemination of scientific research documents, whether they are published or not. The documents may come from teaching and research institutions in France or abroad, or from public or private research centers.
L'archive ouverte pluridisciplinaire HAL, est destinée au dépôt et à la diffusion de documents scientifiques de niveau recherche, publiés ou non, émanant des établissements d'enseignement et de recherche français ou étrangers, des laboratoires publics ou privés. 


\title{
DEFECTS IN CRYSTALS CREATED BY ION BOMBARDMENT : THEORY AND SIMULATION
}

\author{
I. M. TORRENS (*)
}

Theoretical Physics Division, AERE, Harwell, UK

\begin{abstract}
Résumé. - Les processus de dommages créés par un ion énergétique qui pénètre dans un solide cristallin sont traités d'une façon qualitative. Les différentes méthodes d'étudier les mécanismes de dommage et les défauts créés sont décrites, et le rôle de la simulation à l'ordinateur dans la comparaison de la théorie et l'expérience est discuté.
\end{abstract}

\begin{abstract}
The damage processes involved in the penetration of an energetic ion in a crystalline solid are reviewed qualitatively. Different methods of studying the mechanisms of damage and defects created are described and the role of computer simulation in the comparison of theory and experiment is discussed.
\end{abstract}

1. Introduction. - When an energetic heavy ion penetrates the surface of a crystal, it slows down by interaction with the atoms and electrons of the crystal. The damage mechanisms are extremely complicated and not fully understood [1], [2]. Elastic energy transfer processes, electronic excitation and ionisation play roles of varying importance depending on the energy of the incident ion and on the type of solid. Because of the many-body nature of the interaction with the nuclei and electrons it is difficult to formulate a completely reliable theory of the distribution of the energy loss between elastic and inelastic processes. Inelastic loss to electrons can cause damage to insulators and semiconductors, but in the case of metals this energy is largely dissipated as heat without causing any permanent detectable damage. The observable effects occur through the direct or indirect displacement of atoms of the solid from their equilibrium positions. These atoms come to rest as a result of interaction with other atoms and the initial energy of the incident ion is dissipated in a multiple atomic displacement event known as an atomic displacement cascade. When the kinetic energy of the displaced atoms drops to a value comparable with the thermal energy of the undisturbed crystal atoms the final configuration relaxes to a stable damage state which depends on the temperature, background defect concentration and electronic nature of the solid.

In order to estimate the amount of damage it is necessary to study the dynamics of the multiple displacement process during its creation and its partial annealing. For the former it is necessary to know something about the inelastic loss to electrons and

$\left(^{*}\right)$ Now at Energy Division, OECD, Paris $16^{e}$, France. about the elastic interaction potential between the atoms. The latter is governed by the thermal motion of the defects created by displacement, so that their activation energies for formation and migration and the binding energies between different types of defect should be known.

To a certain extent it is possible to treat these problems by analytical means. A number of theories exist for describing the development of a displacement cascade in an amorphous solid, ranging from elementary descriptions [3], [4] to rather involved treatments requiring the use of a computer to solve the integral equations obtained [5]-[7]. So far, however, none of these have been able to take into account either the crystallinity of the material or the manybody nature of the atomic collisions, both of which can be handled quite easily by computer simulation. Similarly, the configuration of point and line defects in crystals may be studied analytically by elasticity theory on the one hand and by electron theory on the other. Both these approaches may be criticised, the former because the theory breaks down close to the defect, and the latter because it does not consider such phenomena as closed shell repulsion between the ions and the relaxation of the lattice about the defect. Again, computer simulation of a section of crystal lattice provides a useful method of studying defect configurations and energies, though this also is subject to criticism, as will be pointed out later.

This review will discuss in mainly qualitative terms the damage mechanisms involved in the creation of defects in a crystal as a result of ion bombardment, and the nature of these defects. The developing role of computer simulation as a theoretical tool will be critically examined in relation to other theoretical methods. 
2. Damage mechanisms. - The rate of energy loss of the ion may be written

$$
\begin{aligned}
\frac{\mathrm{d} E}{\mathrm{~d} x} & =\left(\frac{\mathrm{d} E}{\mathrm{~d} x}\right)_{\text {electronic }}+\left(\frac{\mathrm{d} E}{\mathrm{~d} x}\right)_{\text {nuclear }} \\
& =-\rho_{\mathrm{e}} S_{\mathrm{e}}(E)-\rho_{\mathrm{n}} S_{\mathrm{n}}(E)
\end{aligned}
$$

where $\rho_{\mathrm{e}}, \rho_{\mathrm{n}}$ are electron and nuclear densities of the solid, and $S_{\mathrm{e}}(E), S_{\mathrm{n}}(E)$ the electronic and nuclear stopping powers. This equation is applicable to an amorphous solid of uniform density. The theoretical problem lies in evaluating the stopping-powers. Once these are known, the range of the particle is given by

$$
R=-\int_{0}^{E_{0}} \frac{\mathrm{d} E}{\rho_{\mathrm{e}} S(E)+\rho_{\mathrm{n}} S_{\mathrm{n}}(E)}
$$

The stopping-power is a function of the differential cross-section for varying energy transfers between the incident particle and the electrons or nuclei :

$$
S(E)=\int_{T_{\min }}^{T_{\max }} \frac{T \mathrm{~d} \sigma(E, T)}{\mathrm{d} T} \mathrm{~d} T
$$

Here $T_{\max }$ is the maximum possible energy transfer, and $T_{\min }$ is determined by the threshold for ionisation or atomic displacement. The cross-section $\mathrm{d} \sigma(E, T)$ is a function of the assumed scattering law For example, assuming Rutherford scattering in high energy collisions yields an approximate $1 / E$ dependence for $S(E)$, which is confirmed by more refined quantum mechanical treatments (though the exact equation is altered). At the lower end of the energy scale, treatments of electronic energy loss on the basis of the Thomas-Fermi model of the electron distribution yield a different variation, with $S_{\mathrm{e}}(E) \propto E^{1 / 2}$. This is the range applicable to most ion bombardment experiments.

It is generally assumed, for the purpose of most theoretical treatments, that there is an ionisation threshold $E_{\mathrm{i}}$ below which electronic energy loss of a moving particle is effectively zero. This is normally in the low kilovolt energy range. Some of the simpler theories assume also that the elastic collision loss is zero above $E_{\mathrm{i}}$, in other words that there is a stepfunction change over from elastic to inelastic energy loss. Although quite evidently an over-simplification, this is a useful first approximation and greatly facilitates the elementary theory of multiple collision events. The alternative is to calculate the repartition of energy loss using available expressions for the stopping-powers of eq. (1) [1], [2].

Consideration of defect configurations arising from elastic energy transfer processes hinge on two important parameters, namely the atomic interaction potential and the atomic displacement threshold, which we shall now discuss briefly.

3. The interatomic potential. - In a review such as this, only a very brief qualitative treatment of such an involved subject as the interatomic potential is possible. The topic is again complicated as a result of the many-body interactions, and the potential is approximated by many different analytical forms depending mainly on the relativeenergy of the atoms [8]. Basically, at very high energies the nucleus-nucleus interaction predominates and the potential may be represented by a simple Coulomb form, and at large separations and low energies a Van der Waals polarisation interaction prevails. The region from thermal energies up to the high kilovolt range, which is that of interest to ion bombardment studies, may again be separated into two parts. At the higher energies the atomic electrons may be considered to screen the nuclear interaction, and a screened Coulomb potential is then applicable : this consists of multiplying the Coulomb interaction by one of a number of possible forms of screening function whose effect is to reduce its range. At energies up to $\sim 10 \mathrm{keV}$ the nuclear interaction may be effectively neglected and the potential represented by a Born-Mayer interaction between closed electron shells of the ions with parameters determined from experimental data. There are analytical forms which combine the above two types to provide a potential valid over the whole region of interest.

For studies in the thermal equilibrium energy range, e. g. defect studies in crystals, the potential must contain a cohesive minimum. The depth of this minimum and range of the potential depends on the type of crystal, and the potential parameters are usually found from crystal equilibrium data.

It should be stressed that any potential, analytical or not, is subject to criticism since all two-body potentials represent radical approximations of the many-body interactions between nuclei and electrons. The choice of potential usually depends on the problem under study and the method of analysis. For example, if a purely analytical treatment of the damage is undertaken, a relatively simple form of potential may be necessary, whereas if a computer is used either to solve the integral equations or to simulate the damage, it is possible to handle more sophisticated, and in most cases more realistic, forms of potential.

Some common forms of interatomic potential are illustrated by figure 1 .

4. The displacement threshold. - Theoretically, the displacement threshold energy in a metal or other crystalline material is defined as the minimum kinetic energy which must be given to an atom while on its lattice site, in order for it to be permanently displaced. To move an atom into an interstitial position adjacent to the vacancy requires only a small amount of energy, of the order of a few electron volts. However, when the resulting interstitial loses its kinetic energy to the surrounding atoms it will be in an unstable configuration relative to the vacancy and the result will be an immediate recombination of the unstable pair. For 

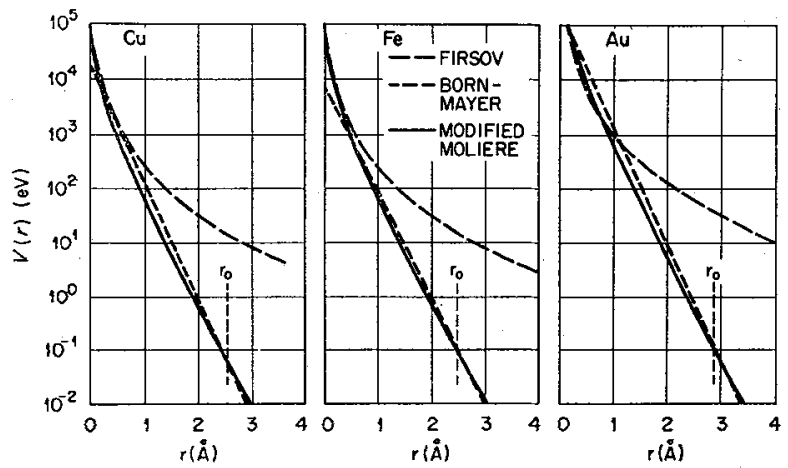

(a)

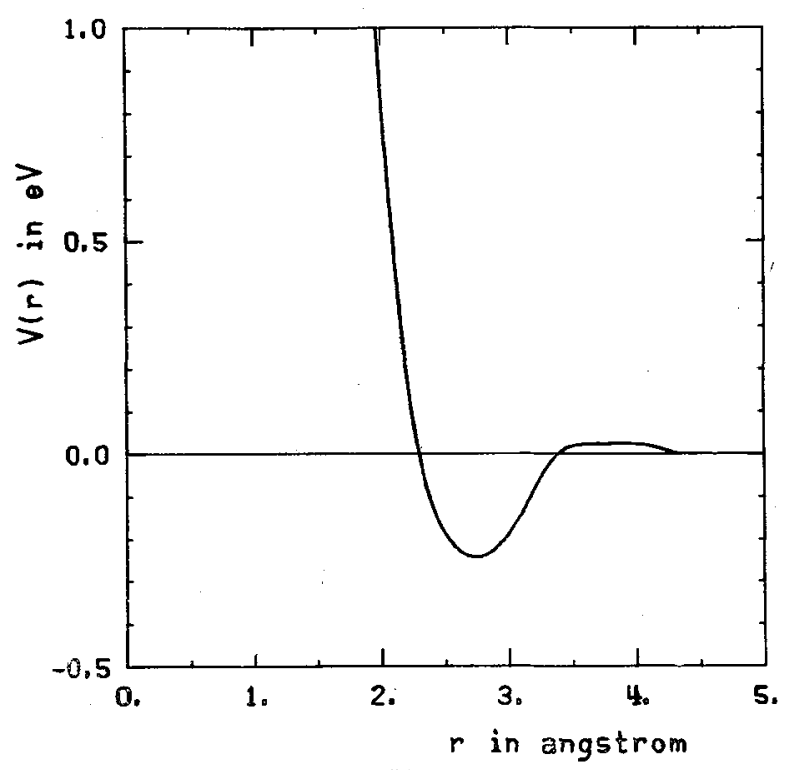

(b)

FIG. 1. - a) Comparison of three pair potentials for atomic collisions in $\mathrm{Cu}, \mathrm{Fe}$ and $\mathrm{Au}$. The screening function of the Molière potential is adjusted so that the potential has the same value as the Born-Mayer potential at the nearest neighbour separation $r_{0} . b$ ) Pair potential for copper constructed from a polynomial representation with parameters adjusted to crystal equilibrium data [31].

permanent displacement to occur it is necessary to separate the interstitial from the vacancy by a distance sufficient to preclude the spontaneous recombination process. Computer simulation studies [9], [10], [17] indicate that the stable configurations depend on lattice geometry, recombination distances being greater along closepacked directions than in other directions. A further problem is that spontaneous recombination is a temperature-sensitive phenomenon. At high temperatures an interstitial close to its original vacancy may migrate freely, overcoming the potential barrier which would otherwise force it to recombine.

Theoretical methods of studying the displacement threshold are limited to either very elementary considerations or to computer simulation techniques. An early estimation, given by Seitz and Koehler [11], was a value about four times the sublimation energy, which for copper gave the much-quoted threshold of $25 \mathrm{eV}$. Experimental results for a number of metals tend to indicate thresholds between $15 \mathrm{eV}$ and $50 \mathrm{eV}$, and confirm their directional dependence [12], [13]. Some recent computer simulation work by the author [13 a] on the dynamics of low energy displacement in copper, suggests that the resulting interstitialvacancy separation for displacement of a given energy is a rather complicated function of direction, which, added to the recombination direction-dependence, renders the picture even more confused.

In the absence of reliable information on the displacement threshold, most theoretical and simulation damage studies assume an isotropic threshold in the region of $25 \mathrm{eV}$. This is more reasonable than it seems at first glance, since we are mainly considering multiple atomic displacement events where the knock-on direction relative to the lattice may be assumed to average out. It is possible however that some displacements in close-packed directions could be neglected when this average value of threshold is used.

5. The atomic displacement cascade. - The cascade of displaced atoms resulting from an incident primary energetic ion involves both elastic and inelastic collisions, though fortunately these two types of collision may be separated to a good approximation. The simplest theory [3] assumes an amorphous solid, purely hard sphere collisions, no thermal effects. an isotropic displacement threshold $E_{\mathrm{d}}$, and an ionisation threshold $E_{\mathrm{i}}$ above which only inelastic loss occurs and below which only elastic collisions occur. This theory gives an expression for the total number of displaced atoms $v(E)$ resulting from an incident ion of energy $E$ :

$$
v(E)=\left\{\begin{array}{cc}
E_{\mathrm{i}} / 2 E_{\mathrm{d}}, & E>E_{\mathrm{i}} \\
E / 2 E_{\mathrm{d}}, & 2 E_{\mathrm{d}}<E<E_{\mathrm{i}} \\
0, & E<2 E_{\mathrm{d}} .
\end{array}\right.
$$

When inelastic collisions are taken into account and the hard-sphere potential is replaced by a softer and more realistic interaction, the solution of the set of integral equations [6], [7] suggests that the number of displacements should be represented by the modified expression :

$$
\vee(E)=\kappa \cdot \frac{E_{\text {damage }}}{2 E_{\mathrm{d}}}
$$

where $E_{\text {damage }}$ is the primary energy less the total inelastic energy loss in all collisions. For the purposes of this theory there is an inelastic energy loss in each collision depending basically on the hardness of the collision and on the electron density of the medium [14], [15]. The constant $\kappa$, known as the displacement efficiency, depends on the interatomic potential, being $\sim 0.8$ for realistic interactions.

The damage energy $E_{\text {damage }}$ in the cascade may be 
estimated using the Lindhard theory of inelastic loss [14], yielding an expression of the form [15] :

$$
E_{\text {damage }}=\frac{E}{1+k g(\varepsilon)}
$$

where $k$ is a constant depending on the mass and charge of the primary, and $g(\varepsilon)$ is a universal function of the primary ion energy, and the mass and charge of both the ion and the atoms of the solid.

Although the analytical theory does not include crystal lattice effects, computer simulations have shown that these are relatively unimportant (see next section) and that an expression such as that of eq. (6) is a good representation of the number of defects to be expected in a displacement cascade from a given energy primary.

6. Simulation of displacement cascades. - The use of the computer to simulate the development of an atomic displacement cascade in a crystal permits us to study the possible effects of the crystal lattice and also the details of the spatial distribution of the defects at the end of the cascade. It is well known that an energetic ion projected along a low-index crystal direction may be channelled between rows or planes in the lattice, thereby undergoing an anomalously low energy loss and greatly increased penetration into the crystal. This is basically a high energy phenomenon since the probability of scattering out of the channel increases as the energy decreases. The other principal directional effect is focusing, or the efficient transfer of energy along a close-packed line of atoms from one atom to the next, resulting in energy being carried out of the immediate neighbourhood of the centre of the cascade. Focusing occurs mainly at low energies, up to a few hundred electron volts, and may or may not be accompanied by a replacement sequence, that is, the successive replacement of one atom in the lattice line by the previous one. The importance of these effects in influencing the development of a displacement cascade may only be studied by computer simulation.

At low energies of the order of $10 \mathrm{eV}$ or less, it is necessary to take into account the interaction of a moving atom with all the atoms in its neighbourhood out to a distance at which the potential is negligibly small. The method is then to simulate a small section of crystallite of some 1000 atoms which interact with an assumed pairwise potential. Then one atom is given the desired energy and the classical equations of motion of all the atoms of the crystal which receive greater than thermal energies are integrated by a difference procedure over successive time increments. In this way the development of the displacement event is followed until all energies have dropped to the thermal range. This method is particularly useful for energies up to a few times the displacement threshold but is rather time-consuming for the kilovolt range. Fortunately, for collision energies above about $10 \mathrm{eV}$ the two-body approximation is valid, since the interatomic potential rises steeply at these energies Then classical two-body scattering equations may be used to follow the development of the displacement. cascade [18]. There is an added advantage in that the computer can create the crystal as the cascade develops and there is no need to store crystal in the memory of the machine. The method is to give the primary knock-on atom, or the incident ion in the case of bombardment, the required energy, and follow the series of two-body collisions of both the primary and its progeny until all energies have dropped below the assumed displacement thresholds. The progeny are those atoms which receive energy greater than the displacement threshold as a result of being struck by a moving atom. The final result consists of a number of vacancies and interstitials in the region of the primary, an example of which is shown in figure 2, representing a $14 \mathrm{keV}$ cascade in $\alpha$-iron [19]. The separation into two or more sub-cascades is typical of cascades from higher energy primaries, and is caused by short-range channelling of secondary atoms in the intervening undamaged crystal, travelling some 10 to 20 lattice parameters without causing further displacements until they are finally dechannelled.

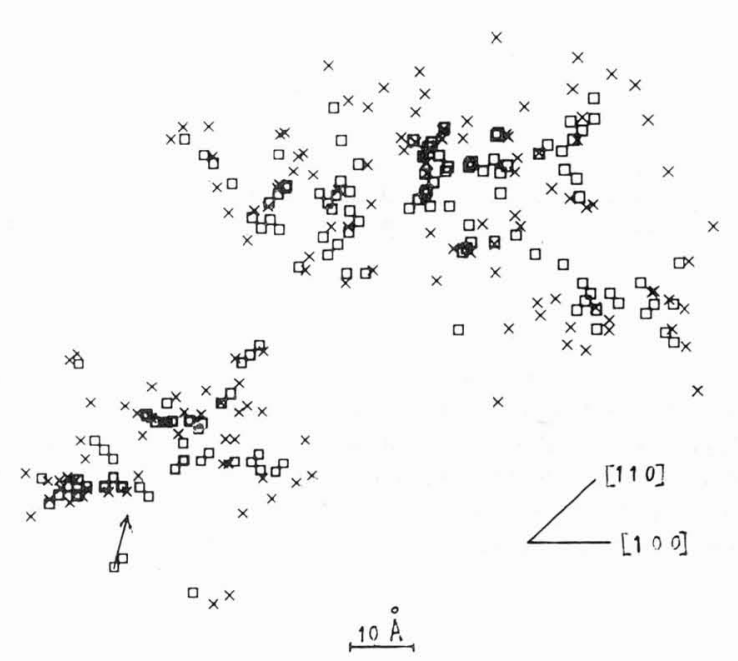

Frg. 2. - Defects resulting from a $14 \mathrm{keV}$ cascade in $\alpha$-Fe. The primary knock-on atom is arrowed. Vacancies are represented by squares and interstitials by crosses. The cascade is projected onto a $\left(\begin{array}{lll}1 & 0 & 0\end{array}\right)$ plane and the principal crystallographic directions are indicated.

A study of a large number of cascades in copper, iron and gold [18] revealed that when the primary originates from a lattice site, the incidence of longrange channelling is negligible, and the number of defects created is very similar to that which would be created in an amorphous solid. The more frequent short-range channelling influences the spatial distribution of the defects, splitting up the cascade into two or more smaller cascades. This could have a significance from the point of view of subsequent 
annealing of the damage, but has no appreciable effect on the number of defects created.

In the case of bombardment of a crystal by energetic ions, if the direction of irradiation is parallel to a low-index crystal direction, channelling of the primary will play a more important role, and the phenomenon has occasionally been used to ensure penetration and avoid surface-related effects. For a channelled particle the result will be increased inelastic and sub-threshold loss along the channelled part of its path, with a resulting smaller number of defects created at the end of its trajectory. Because different ions will be channelled over different distances it is more difficult to calculate the total number of defects created by the ion beam.

Another problem in the case of ion bombardment is related to the proximity of the surface to the cascade created. This will result in escape of some interstitials through the surface either directly following their creation or during the annealing process after the cascade is completed. The effect of this on the final number of defects will vary depending on whether the escaping atom carries with it a high energy or not. Fortunately most high energy atoms are scattered forward and the escaping secondaries generally have quite low energy.

The computer simulation of displacement cascades has led to the suggestion of a standard method of calculating the number of defects caused by a primary of energy $E$ [20]. Since directional effects are found to be insignificant on average, $v(E)$ is given by eq. (6) with $E_{\text {damage }}$ calculated using eq. (7). Because of the uncertainty of the value of $E_{\mathrm{d}}$, it was deemed appropriate to select a single value irrespective of target.

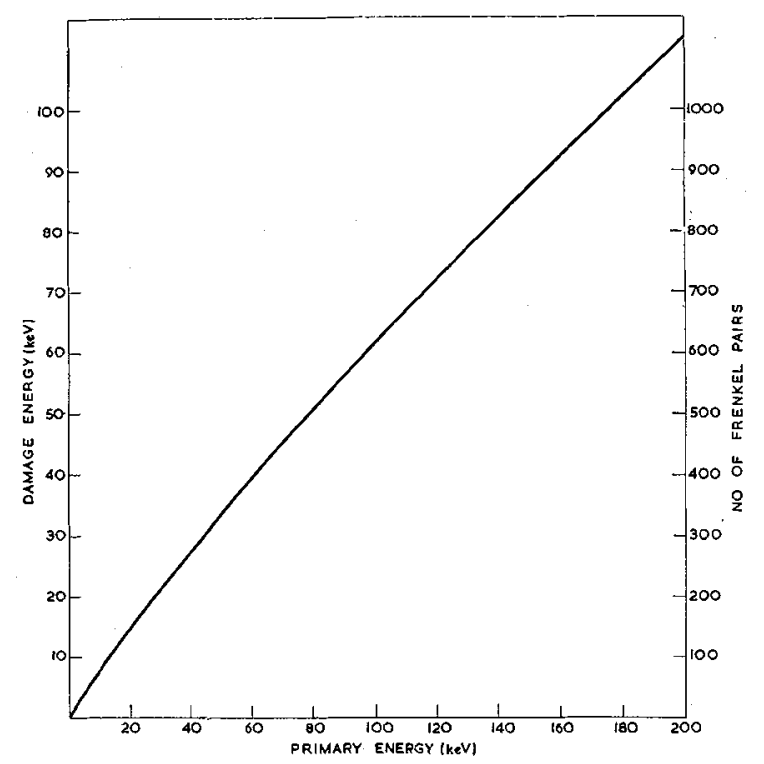

FIG. 3. - Plot of the damage energy and number of Frenkel pairs created in $\alpha$-Fe against primary knock-on energy, according to the damage eq. (8) [20].
If this was assumed to be $40 \mathrm{eV}$, then with $\kappa=0.8$, the number of displacements is:

$$
v(E)=10 E_{\text {damage }}
$$

where $E_{\text {damage }}$ is measured in $\mathrm{keV}$. The function of this proposed standard is to facilitate correlation of results of different irradiation experiments where the total radiation dose is usually given in displacements per atom, and up to now there has been considerable variation in the method of calculation of this unit. It is not meant to be an accurate representation of the number of defects created, although when applied to some reactor materials it gives good agreement with experimental results. Certainly the use of such a standard formula would facilitate comparison of the results of different ion bombardment experiments using accelerators. Figure 3 is a plot of the damage energy and number of Fenkel pairs produced in iron by an incident homonuclear ion of a given energy (assuming no directional or surface effects) using this standard method.

7. Defects created by bombardment. - The basic defects are vacancies and interstitials, but owing to their concentration within the limits of the displacement cascade, they are frequently (especially the vacancies) found in clusters of two or more. This clustering is particularly important in the case of annealing of the cascade, since single, bi-, tri- and higher order vacancies or interstitials have different mobilities and binding energies. Large vacancy clusters tend to be rather stable and immobile and if sufficiently large they can arrange themselves in a dislocation loop, which is their lowest energy configuration. This is also true for interstitial clusters. If highly mobile gaseous impurities are present in the crystal, as they are in reactor materials, they can be trapped in a quite small vacancy cluster and stabilise it, inhibiting loop formation and enabling it to grow larger if the temperature and vacancy mobility are within a suitable range [21]. This is the theory behind the formation of voids in highly irradiated metals particularly under reactor conditions. In particular, ion bombardment experiments using accelerators have demonstrated that pre-injection with helium greatly enhances the amount of void formation occurring during heavy ion irradiation [22], [23]. The presence of displacement cascades is a sufficient, but not necessary condition for void formation, since a high defect concentration will suffice to nucleate voids, as high voltage electron microscope irradiation has demonstrated [24], [25]. Although of course an equal number of interstitials and vacancies is generated in an irradiation, provided the vacancies are sufficiently mobile the interstitials have a preference for dislocation sinks and loop formation.

The problem of annealing of a high defect concentration is one of many-body kinetics, and may be approached using either kinetic theory of the defects 
assumed to be in a uniform infinite medium [26] or by a Monte Carlo simulation following the directed random walk process of each defect under the influence of other nearby defects [27]. Both of these methods require as input some information on the mobility of single and multiple defects and on the binding energy of defect clusters. Some information may be obtained from experiment, but it is frequently difficult (and often a question of interpretation of the results) to isolate the required parameters within a given experiment. Defect properties may also be studied by theoretical methods [28] and by simulation. In fact the comparison of simulation with experimental tesults for quenching and electron irradiation has been useful in determining some defect mobilities and binding energies [29]. We shall now outline the simulation method as applied to defect energies.

8. Crystal defect simulations. - The purpose of these calculations is generally to find the lattice relaxation and potential energy associated with one or more defects in a crystal. In the volume of lattice surrounding the defect which is permitted to relax the atoms are free to move under the influence of pairwise interatomic potentials. Outside this volume the atoms are normally either held fixed or assumed to be slightly displaced according to their elastic interaction with the defect. If for example the energy of migration of a point defect is required, the lattice is relaxed first with the defect at its equilibrium position, then with the migrating atom at the saddlepoint for the jump. The difference in potential energy of these two configurations is the activation energy for the jump. Binding energies are found similarly by relaxation with the two defects first completely separate, then together. To find the energy of formation an additional contribution to the lattice energy arises from the volume change, and this must be calculated quantum mechanically. Lattice relaxation simply consists of minimising the potential energy in the relaxed volume using one of a number of available algorithms [19].

The methods described above apply to a static lattice, and neglect the effects of thermal vibration. This may be taken into account using the technique of molecular dynamics, which is basically that described for low energy cascades in section 6 , with the difference that all atoms of the crystallite are assumed to move, and periodic boundary conditions are imposed to ensure energy conservation. The method is then to study the dynamics of the migration process and hence from the temperature and lattice characteristics, to estimate the jump activation energy. This method, while more rigorous than the static lattice method, is enormously time-consuming, since the movement of all atoms must be followed through hundreds of vibration periods in order to have a sufficient number of jumps for reasonable statistics. For this reason it has not achieved much popularity.
One problem related to all defect simulations lies in the application of a pairwise interatomic potential to the atoms in the immediate neighbourhood of a lattice defect. Even assuming the absence of higherorder interactions between the atoms, most pair potentials contain parameters adjusted to crystal equilibrium data, and are therefore applicable in principle only to a perfect periodic lattice. The details of an assumed interaction may well be altered in the vicinity of a vacancy or other defect, and it is difficult to estimate to what extent this influences the computed results. It is in fact well established that the numerical results of static defect simulations are rather potential-sensitive. The value of defect simulations therefore seems to lie in comparative values for two or more quantities rather than absolute numerical values for a given parameter - for example in comparing the single, di- and tri-vacancy migration energies, the relative magnitudes are likely to be much less sensitive to the potential. Thus if the experimental single vacancy migration energy is known, those of the di- and tri-vacancies may be inferred from the simulation.

9. Interpretation of computer simulation results. Computer simulations have frequently been referred to as " computer experiments". The difference between these and real experiments if of course that in the computer the laws of nature are provided as input data to as good an approximation as possible, based on current knowledge and machine limitations. It cannot be sufficiently stressed that the results of computer simulations, like those of any other theoretical study, are only as good as the models. Early simulations tended to over-emphasise the numerical results and neglect the essential sensitivity analysis, with consequent adverse effects on the image of computer simulation work in the radiation damage and defects field. This, however, is no reason for abandoning what is in effect a very powerful theoretical tool, whose potential is being increasingly realised, especially when it is used in comparison with experiment. Provided that a certain amount of caution is exercised in interpreting the results of computer simulations in terms of reality, there is no reason why these techniques should not be used with considerable success to improve our understanding of problems involving atomic motion and defects in crystals.

10. Conclusion. - The purpose of this article has been to review very qualitatively the theoretical aspects of damage mechanisms and defects produced by energetic ion bombardment of crystalline solids. Apologies are made for any incompleteness evident to those with an intimate knowledge of the field.

One field which has not been treated here is that of the formation of junctions in semiconducting 
crystals by ion injection. The basic damage mechanisms are similar, though there the emphasis is on alteration of the electronic properties of the solid. There is a burgeoning literature on the subject and good reviews are available [30].

The most important conclusion of recent experimental and theoretical studies of ion bombardment is that advances in the understanding of damage and defects created by irradiation require the close cooperation of theorists and experimentalists. This is particularly true of computer simulation work, where comparison with experiment can be extremely valuable in determining many of the parameters which form the basis of the computer models, and where the feedback can frequently lead to a better interpretation of experimental results.

\section{References}

[1] Thompson, M. W., Defects and Radiation Damage in Metals (Cambridge University Press, London) 1969.

[2] Chadderton, L. T., Radiation Damage in Crystals (Methuen London) 1965.

[3] Kinchin, G. H. and Pease, R. C., Rept. Progr. Phys. 18 (1955) 1

[4] SNyder, W. S. and Neufeld, J., Phys. Rev. 97 (1955) 1636.

[5] Sigmund, P., Rad. Eff. 1 (1969) 15.

[6] Winterbon, K. B., Stgmund, P. and Sanders, J. B., Kgl. Danske Vidensk. Selsk. Mat. Fys. Medd. 37 (1970) No. 14.

[7] Robinson, M. T., Phil. Mag. 17 (1968) 639.

[8] Torrens, I. M., Interatomic Potentials (Academic Press, New York) 1972.

[9] Erginsoy, G., Vineyard, G. H. and Englert, A., Phys. Rev. A 133 (1964) 595.

[10] Johnson, R. A., Rad. Eff. 2 (1969) 1.

[11] Seitz, F. and Koenler, J. S., Solid State Physics 2, ed. Seitz and Turnbull (Academic Press, New York) 1957 , p. 305.

[12] Sosin, A. and Bauer, W., Studies in Radiation Effects in Solids 3, ed. Dienes (Gordon and Breach, New York) p. 153.

[13] Jung, P. and Schilling, W., Phys. Rev. B 5 (1972) 2046.

[13 a] Torrens, I. M., J. Phys. F (1973) in press.

[14] Lindhard, J., Nielson, V., Scharff, M. and Thomsen, P. V., Kgl. Danske Vidensk. Selsk. Mat. Fys. Medd. 33 (1963) No. 10.

[15] FIRsov, O. B., Sov. Phys. JETP 36 (1959) 1517.

[16] Robinson, M. T., Nuclear Fusion Reactors (British Nuclear Energy Society, London) 1970, p. 364.
[17] Gibson, J. B., Goland, A. N., Milgram, M. and VineYARD, G. H., Phys. Rev. 120 (1960) 1229.

[18] Torrens, I. M. and Robinson, M. T., Interatomic Potentials and Simulation of Lattice Defects ed. Gehlen, Beeler and Jaffee (Plenum Press, New York) 1972, p. 423.

[19] Torrens, I. M., Computer Phys. Comm. 5 (1973) 32.

[20] Norgett, M. J., Robinson, M. T. and Torrens, I. M., CEA (France) Report No. CEA-R-4389 (1972).

[21] Bullough, R. and Nelson, R. S., to be published.

[22] Nelson, R. S., Mazey, D. T. and Hudson, J. A., J. Nucl. Mat. 37 (1970) 1.

[23] Glowinski, L., Fiche, C. and Lotte, M., J. Nucl. Mat. 47 (1973) 295.

[24] NoRrIs, D. I. R., J. Nucl. Mat. 40 (1971) 66.

[25] MAKIN, M. J., Voids Formed by Irradiation of Reactor Materials, ed. Pugh, Loretto and Norris, to be published.

[26] Brailsford, A. D. and Bullough, R., J. Nucl. Mat. 44 (1972) 121.

[27] LANore, J. M., CEA (France), Note No. CEA-N-1567 (1972).

[28] QuÉRÉ, Y., Défauts Ponctuels dans les Métaux (Masson, Paris) 1967.

[29] Lévy, V., Hillairet, J. and Lanore, J. M., Phil. Mag. (in press).

[30] Radiation Damage and Defects in Semiconductors, ed. Whitehouse (Institute of Physics, Conference Series 16) 1973.

[31] Englert, A., Tompa, H. and Bullough, R., Fundamental Aspects of Dislocation Theory (NBS Special Publication 317) 1971, p. 273. 